\title{
On the subpartitions of the ordinary partitions, II
}

\author{
Byungchan Kim* \\ School of Liberal Arts \\ Seoul National University of Science and Technology \\ Seoul, Republic of Korea \\ bkim4@seoultech.ac.kr \\ Eunmi Kim \\ Center for Applications of Mathematical Principles \\ National Institute for Mathematical Sciences \\ Daejeon, Republic of Korea \\ ekim@nims.re.kr
}

Submitted: Jun 30, 2013; Accepted: Oct 21, 2014; Published: Oct 30, 2014

Mathematics Subject Classifications (2010): 05A17, 11P82

\begin{abstract}
In this note, we provide a new proof for the number of partitions of $n$ having subpartitions of length $\ell$ with gap $d$. Moreover, by generalizing the definition of a subpartition, we show what is counted by $q$-expansion

$$
\prod_{n=1}^{\infty} \frac{1}{1-q^{n}} \sum_{n=0}^{\infty}(-1)^{n} q^{\left(a n^{2}+b n\right) / 2}
$$

and how fast it grows. Moreover, we prove there is a special sign pattern for the coefficients of $q$-expansion

$$
\prod_{n=1}^{\infty} \frac{1}{1-q^{n}}\left(1-2 \sum_{n=0}^{\infty}(-1)^{n} q^{\left(a n^{2}+b n\right) / 2}\right) .
$$
\end{abstract}

Keywords: partition; subpartition; partial theta function.

*This research has been supported by TJ Park Science Fellowship of POSCO TJ Park Foundation. 


\section{Introduction}

Let $a_{1} \geqslant a_{2} \geqslant \cdots \geqslant a_{m}$ be an ordinary partition [1]. In a recent paper [9], the first author defines a subpartition of an ordinary partition as follows. Let us fix a positive integer $d$. Then, for a given partition, a subpartition with gap $d$ is defined as the longest sequence satisfying $a_{1}>a_{2}>\cdots>a_{s}$ and $a_{s}>a_{s+1}$, where $a_{i}-a_{j} \geqslant d$ for all $i<j \leqslant s$. $a_{s+1}$ must be understood as a zero if it comes after the final part. This is a generalization of L. Kolitsch's Rogers-Ramanujan subpartition [10], which is the case $d=2$. We call the first condition involving $d$ a gap condition and the second condition $a_{s}>a_{s+1}$ a tail condition. For convenience, we define the subpartition of the empty partition as the empty partition. We define the length of the subpartition with gap $d$ as the number of parts in the subpartition. When the gap $d$ is clear in the context, we will write "the subpartition" instead of "the subpartition with gap d". In [9], the author uses subpartitions to find combinatorial proofs of entries in Ramanujan's lost notebook [11]. Moreover, these subpartitions play a crucial role in obtaining an asymptotic formula for certain $q$-series involving partial theta functions [8].

Define $p(n)$ to be the number of partitions of $n$ and $p(n, \ell, d)$ to be the number of partitions of $n$ having a subpartition of length $\ell$ with gap $d$. In [9], by finding a generating function via a case by case argument, the first author proved that

Theorem 1. For all nonnegative integers $n$ and $\ell$ and a positive integer $d$,

$$
p(n, \ell, d)=p\left(n-S_{\ell, d}\right)-p\left(n-S_{\ell+1, d}\right)
$$

where, for each nonnegative integer $k$,

$$
S_{k, d}=\left\{\begin{array}{l}
1+(1+d)+(1+2 d)+\cdots+1+(k-1) d=\frac{d k^{2}-(d-2) k}{2}, \text { if } k \neq 0, \\
0, \text { if } k=0 .
\end{array}\right.
$$

Example 2. According to Theorem 1, there are 5 partitions of 8 having a subpartition of length 2 with gap 2 as $p(8,2,2)=p(8-4)-p(8-9)=p(4)-p(-1)=5-0=5$. Here are 5 such partitions and the parts consisting of the subpartition are underlined:

$$
\underline{7}+\underline{1}, \underline{6}+\underline{2}, \underline{5}+\underline{3}, \underline{5}+\underline{2}+1, \text { and } \underline{4}+\underline{2}+1+1 \text {. }
$$

In this note, by employing a combinatorial argument, we give a simpler proof.

Now we further generalize the notion of subpartitions as follows. We introduce a new parameter $t$ and replace the tail condition by $a_{s}-a_{s+1} \geqslant t$. The case $t=1$ is the original definition of a subpartition with gap $d$. Now we define $p(n, \ell, d, t)$ to be the number of partitions of $n$ having a subpartition of length $\ell$ with gap $d$ and tail condition $t$. Then, by employing essentially same argument, we can prove the following theorem.

Theorem 3. For all nonnegative integers $n$ and $\ell$, and positive integers $d$ and $t$,

$$
p(n, \ell, d, t)=p\left(n-T_{\ell, d, t}\right)-p\left(n-T_{\ell+1, d, t}\right),
$$


where, for each nonnegative integer $k$,

$$
T_{k, d, t}=\left\{\begin{array}{l}
t+(t+d)+(t+2 d)+\cdots+t+(k-1) d=\frac{d k^{2}+(2 t-d) k}{2}, \text { if } k \neq 0, \\
0, \text { if } k=0 .
\end{array}\right.
$$

By summing even $\ell$ 's, we see that, for a positive integer $a$ and an integer $b$ with $a+b>0$ and $a \equiv b(\bmod 2)$, we find that

$$
\frac{1}{(q ; q)_{\infty}} \sum_{n=0}^{\infty}(-1)^{n} q^{\left(a n^{2}+b n\right) / 2}=\sum_{n=0}^{\infty} p_{e}(n, a,(a+b) / 2) q^{n}
$$

where $(q ; q)_{\infty}=\prod_{n=1}^{\infty}\left(1-q^{n}\right)$ and $p_{e}(n, d, t)$ is the number of partitions of $n$ having subpartitions of even length with gap $d$ and tail condition $t$. Here the assumption on $a$ and $b$ is for the positive integrality of $\left(a n^{2}+b n\right) / 2$ for all positive integers $n$. From the representation of the partial theta function on the left side of (1), it is not clear at all the positivity of its $q$-expansion and what it counts. Since $n$ copies of 1 is always counted by $p_{e}(n, a,(a+b) / 2)$, the positivity of $q$-expansion is now clear from the combinatorial description. The case $a=b=1$ appears Andrews [2] as a generating function for the number of partitions of $n$ in which the first non-occurrence number as a part is odd, which is a conjugation of partition with subpartition of even length with gap $d$ as noted in [8]. When $a=1$ and $b=3$, we have

$$
\frac{1}{(q ; q)_{\infty}} \sum_{n=0}^{\infty}(-1)^{n} q^{\left(n^{2}+3 n\right) / 2}=1+q+q^{2}+2 q^{3}+3 q^{4}+5 q^{5}+\cdots .
$$

Among 7 partitions of 5 , there are 5 partitions having subpartitions of even length with gap 1 with tail condition 2 as follows:

$$
4+1,3+2,2+2+1,2+1+1+1, \quad 1+1+1+1+1 .
$$

Moreover, by adopting the argument in [8], we can prove the following theorem.

Theorem 4. As $n$ tends to infinity, for positive integers $d$ and $t$,

$$
p_{e}(n, d, t) \sim \frac{1}{2} p(n) .
$$

This is a generalization of [8, Theorem 1] and says that asymptotically half of the partitions of $n$ have subpartitions of even length. Much less obviously, there are inequalities between $p_{e}(n, a,(a+b) / 2)$ and $p_{o}(n, a,(a+b) / 2)$, where $p_{o}(n, a,(a+b) / 2)=$ $p(n)-p_{e}(n, a,(a+b) / 2)$, i.e. the number of partitions of $n$ having subpartitions of odd length with gap $a$ and tail condition $(a+b) / 2$. These inequalities are unexpected since both $p_{e}(n, a,(a+b) / 2)$ and $p_{o}(n, a,(a+b) / 2)$ are asymptotically $p(n) / 2$. 
Theorem 5. For integers $a$ and $b$ satisfying $a>0, a+b>0$, and $a \equiv b(\bmod 2)$, we have

$$
\begin{array}{ll}
p_{e}(n, a,(a+b) / 2)>p_{o}(n, a,(a+b) / 2), & \text { if } b>0, \\
p_{e}(n, a,(a+b) / 2)<p_{o}(n, a,(a+b) / 2), & \text { if } b<0,
\end{array}
$$

for large enough integers $n$. Moreover, for $b=0$ and even integers $a>2$, we have

$$
p_{e}(n, a, a / 2)>p_{o}(n, a, a / 2),
$$

for all positive integers $n$ except that the equality holds when $n=2$ and $a=4$.

Remark 6 . The case $a=2$ and $b=0$ was discussed in [8, Theorem 2]. In this case, the sign of $p_{e}(n, 2,1)-p_{o}(n, 2,1)$ is alternating. This difference is due to that the generating function is essentially modular in this case. The more precise statement in the second part is also due to that $1-2 \sum_{n=0}^{\infty}(-1)^{n} q^{\left(a n^{2}+b n\right) / 2}$ becomes a theta function in these cases.

Remark 7 . The conditions on $a$ and $b$, i.e. $a>0, a+b>0$, and $a \equiv b(\bmod 2)$, are needed just for having non-negative integer exponents in the $q$-expansion.

This paper is organized as follows. In Section 2, we prove the combinatorial results. By adopting the circle method and elementary $q$-series manipulation, we will prove Theorem 5 in Section 3.

\section{Proof of Combinatorial Results}

For a given partition $\lambda$, we always write it in the form $\lambda_{1} \geqslant \lambda_{2} \geqslant \cdots \geqslant \lambda_{m}$, and for convenience, we define $\lambda_{s}=0$ for all integer $s>m$. It is well known [1] that

$$
\sum_{n=0}^{\infty} p(n) q^{n}=\frac{1}{(q ; q)_{\infty}} .
$$

Now we define $p(n, t, d)$ to be the number of partitions of $n$ having subpartitions of length $\geqslant m$ with gap $d$. Then, the following lemma immediately implies Theorem 1 .

Lemma 8. For all nonnegative integers $m$,

$$
p(n, m, d)=p\left(n-S_{m, d}\right) .
$$

Proof. It is enough to show that

$$
\sum_{n=0}^{\infty} p(n, m, d) q^{n}=\frac{q^{S_{m, d}}}{(q ; q)_{\infty}} .
$$

By definition, it is clear that the above holds when $t=0$, and thus we may assume that $t \geqslant$ 1. We first observe that $q^{S_{m, d}}$ generates the partition $\pi=(1+(t-1) d, 1+(t-2) d, \ldots, 1)$. 
Let $\lambda$ be a partition generated by $\frac{1}{(q)_{\infty}}$. We append each part of $\lambda$ to $\pi$ beginning with the largest part, and denote the resulting partition as $\mu$, i.e., $\mu_{i}=\pi_{i}+\lambda_{i}$ for all positive integers $i$. For example, when $\pi=(5,3,1)$ and $\lambda=(4,4,3,2,1)$, we obtain $\mu=(9,7,4,2,1)$. Since the gap between two consecutive parts of $\pi$ is larger than or equal to $d$ and $\mu_{m}>\mu_{m+1}$, we see that $\mu$ has a subpartition of length at least $t$, which completes the proof.

By employing the same argument, we can easily see that $\frac{q^{T} m, d, t}{(q ; q)_{\infty}}$ is a generating function for the number of partitions of $n$ having subpartitions of length $\geqslant m$ with gap $d$ and tail condition $t$, which implies Theorem 3.

Now we turn to the proof of Theorem 4. To this end, we are going to employ Ingham's Tauberian theorem ([7, Theorem 1] and [4, Theorem 5.3]). To apply the Tauberian theorem, we have to show that $p_{e}(n, d, t)$ is weakly increasing. To see this, suppose that $\lambda$ is a partition of $n$ with subpartition of even length. If there is no subpartition in $\lambda$, we add a part 1 to the partition $\lambda$. Then, the resulting partition is a partition of $n+1$, and since the size of the first two parts remains the same, the length of the subpartition is 0 . If $\lambda$ contains the subpartition, we increase the largest part of $\lambda$ by 1 . Then, the resulting partition is a partition of $n+1$ and this operation does not affect the length of the subpartition. It is clear that this map is an injection, thus we observe that $p_{e}(n, d, t) \leqslant p_{e}(n+1, d, t)$. Theorem 4 now immediately follows from [3, Theorem 1] and Ingham's Tauberian theorem.

\section{Proof of Theorem 5}

For a positive integer $a$ and an integer $b$ with $a+b>0$ and $a \equiv b(\bmod 2)$, define

$$
\begin{gathered}
S_{a, b}(q):=1+2 \sum_{n=1}^{\infty}(-1)^{n} q^{\left(a n^{2}+b n\right) / 2}, \\
F_{a, b}(q)=\sum_{N=0}^{\infty} \alpha_{a, b}(N) q^{N}:=\frac{1}{(q ; q)_{\infty}} S_{a, b}(q) .
\end{gathered}
$$

Note that $\alpha_{a, b}(N)=p_{e}(N, a,(a+b) / 2)-p_{o}(N, a,(a+b) / 2)$. Therefore, to prove Theorem 5 , it suffices to see the sign of $\alpha_{a, b}(N)$. We are going to get an asymptotic formula for $\alpha_{a, b}(N)$ by similar argument of Bringmann and Mahlburg [4]. Main idea of the proof is that we can get an asymptotic formula by focusing on asymptotic behavior of $S_{a, b}(q)$ near $q=1$.

Set $q=e^{2 \pi i \tau}$ with $\tau=x+i y$. The following proposition describes an asymptotic behavior of $S_{a, b}(q)$ near $q=1$.

Proposition 9. Assume $|x| \leqslant y$. As $y \rightarrow 0+$,

$$
S_{a, b}(q)=\frac{b}{4}(-2 \pi i \tau)+\mathcal{O}\left(y^{2}\right)
$$


To prove this result, we need the following Zagier's result on asymptotic expansions for series (the first generalization of Proposition 3 in [12] with a correction on the sign),

Lemma 10. Suppose that $h$ has the asymptotic expansion

$$
h(t)=\sum_{n=0}^{S} b_{n} t^{n}+\mathcal{O}\left(t^{S+1}\right)
$$

as $t \rightarrow 0+$ and that $h$ and all of its derivatives are of rapid decay at infinity, i.e. $\int_{l}^{\infty}\left|h^{(k)}(x)\right| d x$ converges for some $l>0$. Then, for $a>0$, as $t \rightarrow 0+$,

$$
\sum_{m=0}^{\infty} h((m+a) t)=\frac{1}{t} \int_{0}^{\infty} h(x) d x-\sum_{n=0}^{S} b_{n} \frac{B_{n+1}(a)}{n+1} t^{n}+\mathcal{O}\left(t^{S+1}\right),
$$

where $B_{n}(x)$ is the $n$-th Bernoulli polynomial.

Proof of Proposition 9. Let $f_{a, b}(\tau)=\left(S_{a, b}(q)-1\right) / 2$, i.e.

$$
f_{a, b}(\tau)=\sum_{n=1}^{\infty}(-1)^{n} q^{\left(a n^{2}+b n\right) / 2} .
$$

We can rewrite $f_{a, b}(\tau)$ as follows:

$$
f_{a, b}(\tau)=e^{-\frac{b^{2}}{4 a} \pi i \tau} g_{a, b}(\tau)
$$

where

$$
g_{a, b}(\tau)=\sum_{n=0}^{\infty}\left[e^{4 \pi a\left(n+1+\frac{b}{4 a}\right)^{2} i \tau}-e^{4 \pi a\left(n+\frac{1}{2}+\frac{b}{4 a}\right)^{2} i \tau}\right] .
$$

We will find asymptotic formulas for the real and imaginary parts of $g_{a, b}(\tau)$. The real part of $g_{a, b}(\tau)$ can be written as

$$
\operatorname{Re}\left(g_{a, b}(\tau)\right)=\sum_{n=0}^{\infty}\left[u_{\frac{x}{y}}\left(\left(n+1+\frac{b}{4 a}\right) \sqrt{y}\right)-u_{\frac{x}{y}}\left(\left(n+\frac{1}{2}+\frac{b}{4 a}\right) \sqrt{y}\right)\right],
$$

where

$$
u_{s}(t)=e^{-4 \pi a t^{2}} \cos \left(4 \pi a s t^{2}\right)=1-4 \pi a t^{2}+\mathcal{O}\left(t^{4}\right) \text { as } t \rightarrow 0+.
$$

By Lemma 10, for $\frac{b}{4 a}+\frac{1}{2}>0$ (this is the case as $a>0, a+b>0$ ),

$$
\begin{aligned}
\operatorname{Re}\left(g_{a, b}(\tau)\right)= & {\left[\frac{I_{u}}{\sqrt{y}}-B_{1}\left(1+\frac{b}{4 a}\right)-(-4 \pi a) \frac{B_{3}\left(1+\frac{b}{4 a}\right)}{3} y+\mathcal{O}_{\frac{x}{y}}\left(y^{2}\right)\right] } \\
& -\left[\frac{I_{u}}{\sqrt{y}}-B_{1}\left(\frac{1}{2}+\frac{b}{4 a}\right)-(-4 \pi a) \frac{B_{3}\left(\frac{1}{2}+\frac{b}{4 a}\right)}{3} y+\mathcal{O}_{\frac{x}{y}}\left(y^{2}\right)\right] \\
= & -\frac{1}{2}+\frac{b(2 a+b)}{8} \pi y+\mathcal{O}_{\frac{x}{y}}\left(y^{2}\right)
\end{aligned}
$$


with $\left|I_{u}\right|=\left|\int_{0}^{\infty} u_{s}(t) d t\right|<\infty$. The imaginary part can be treated similarly.

$$
\begin{aligned}
& \operatorname{Im}\left(g_{a, b}(\tau)\right) \\
& =\left[\frac{I_{v}}{\sqrt{y}}-(4 \pi a) \frac{B_{3}\left(1+\frac{b}{4 a}\right)}{3} x+\mathcal{O}_{\frac{x}{y}}\left(y^{2}\right)\right]-\left[\frac{I_{v}}{\sqrt{y}}-(4 \pi a) \frac{B_{3}\left(\frac{1}{2}+\frac{b}{4 a}\right)}{3} x+\mathcal{O}_{\frac{x}{y}}\left(y^{2}\right)\right] \\
& =-\frac{b(2 a+b)}{8} \pi x+\mathcal{O}_{\frac{x}{y}}\left(y^{2}\right)
\end{aligned}
$$

where $v_{s}(t)=e^{-4 \pi a t^{2}} \sin \left(4 \pi a s t^{2}\right)$ and $\left|I_{v}\right|=\left|\int_{0}^{\infty} v_{s}(t) d t\right|<\infty$. Together with the assumption $|x| \leqslant y$, we get

$$
g_{a, b}(\tau)=-\frac{1}{2}-\frac{b(2 a+b)}{8 a}(\pi i \tau)+\mathcal{O}\left(y^{2}\right) .
$$

Therefore, by considering the Taylor expansion of $e^{-\frac{b^{2}}{4 a} \pi i \tau}$,

$$
\begin{aligned}
f_{a, b}(\tau) & =\left(1-\frac{b^{2}}{4 a} \pi i \tau+\mathcal{O}\left(y^{2}\right)\right)\left(-\frac{1}{2}-\frac{b(2 a+b)}{8 a}(\pi i \tau)+\mathcal{O}\left(y^{2}\right)\right) \\
& =-\frac{1}{2}+\frac{b}{8}(-2 \pi i \tau)+\mathcal{O}\left(y^{2}\right)
\end{aligned}
$$

as $y \rightarrow 0+$ with $|x| \leqslant y$.

Next, we consider the behavior of $S_{a, b}(q)$ away from $q=1$.

Proposition 11. For $y=\frac{1}{2 \sqrt{6 N}}$ with $N>0$ and $y \leqslant|x| \leqslant \frac{1}{2}$, we have

$$
\left|S_{a, b}(q)\right| \ll N^{1 / 2}
$$

Proof. For $a>0$ and $a+b>0$, bounding each term in $S_{a, b}(q)$ gives

$$
\left|S_{a, b}(q)\right| \leqslant 1+2 \sum_{n=1}^{\infty}|q|^{n / 2} \ll N^{1 / 2} .
$$

The following two corollaries describe the behavior of the generating function $F_{a, b}(q)$ near $q=1$ and away from $q=1$, respectively.

Corollary 12. Assume $y=\frac{1}{2 \sqrt{6 N}}$ and $|x| \leqslant y$. As $N \rightarrow \infty$,

$$
F_{a, b}(q)=\left(\frac{b}{4}\right) \frac{e^{\frac{\pi i}{12 \tau}}}{\sqrt{2 \pi}}(-2 \pi i \tau)^{3 / 2}+\mathcal{O}\left(N^{-5 / 4} e^{\pi \sqrt{\frac{N}{6}}}\right) .
$$


Proof. From the asymptotic expansion (3.8) in [4]

$$
\frac{1}{(q ; q)_{\infty}}=\sqrt{-i \tau} e^{\frac{\pi i}{12 \tau}}\left(1+\frac{2 \pi i \tau}{24}+\mathcal{O}\left(N^{-1}\right)\right)
$$

we derive

$$
\begin{aligned}
\frac{1}{(q ; q)_{\infty}} S_{a, b}(q) & \left.=\sqrt{-i \tau} e^{\frac{\pi i}{12 \tau}}\left(1+\frac{2 \pi i \tau}{24}+\mathcal{O}\left(N^{-1}\right)\right)\left(\frac{b}{4}(-2 \pi i \tau)+\mathcal{O}\left(N^{-1}\right)\right)\right) \\
& =\sqrt{-i \tau} e^{\frac{\pi i}{12 \tau}}\left(\frac{b}{4}(-2 \pi i \tau)+\mathcal{O}\left(N^{-1}\right)\right)
\end{aligned}
$$

by combining with Proposition 9 .

Corollary 13. If $y=\frac{1}{2 \sqrt{6 N}}$ with $N>0$ and $y \leqslant|x| \leqslant \frac{1}{2}$,

$$
\left|F_{a, b}(q)\right| \ll e^{\pi \sqrt{\frac{N}{6}}-\frac{\sqrt{6 N}}{5 \pi}} .
$$

Proof. Proposition 11 together with the bound from [5, Lemma 3.5]

$$
\frac{1}{\left|(q ; q)_{\infty}\right|} \ll \sqrt{y} \exp \left[\frac{1}{y}\left(\frac{\pi}{12}-\frac{1}{2 \pi}\left(1-\frac{1}{\sqrt{2}}\right)\right)\right]
$$

gives the corollary.

Now, we use the Circle Method with the results on $F_{a, b}(q)$ to see the sign pattern of $\alpha_{a, b}(N)$. By Cauchy's Theorem, we find

$$
\alpha_{a, b}(N)=\frac{1}{2 \pi i} \int_{\mathcal{C}} \frac{F_{a, b}(q)}{q^{N+1}} d q=\int_{-1 / 2}^{1 / 2} F_{a, b}\left(e^{-\frac{\pi}{\sqrt{6 N}}+2 \pi i x}\right) e^{\pi \sqrt{\frac{N}{6}}-2 \pi i N x} d x
$$

where $\mathcal{C}=\left\{|q|=e^{-\frac{\pi}{\sqrt{6 N}}}\right\}$. We separate this integral into two integrals,

$$
I^{\prime}=\int_{|x| \leqslant \frac{1}{2 \sqrt{6 N}}} F_{a, b}\left(e^{-\frac{\pi}{\sqrt{6 N}}+2 \pi i x}\right) e^{\pi \sqrt{\frac{N}{6}}-2 \pi i N x} d x
$$

and

$$
I^{\prime \prime}=\int_{\frac{1}{2 \sqrt{6 N}} \leqslant|x| \leqslant \frac{1}{2}} F_{a, b}\left(e^{-\frac{\pi}{\sqrt{6 N}}+2 \pi i x}\right) e^{\pi \sqrt{\frac{N}{6}}-2 \pi i N x} d x .
$$

The first integral $I^{\prime}$ gives the main contribution to the coefficient $\alpha_{a, b}(N)$.

Proposition 14. As $N \rightarrow \infty$,

$$
I^{\prime}=b \frac{\pi^{2}}{24 \sqrt[4]{24}} N^{-5 / 4} I_{-5 / 2}\left(\pi \sqrt{\frac{2 N}{3}}\right)+\mathcal{O}\left(N^{-7 / 4} e^{\pi \sqrt{\frac{2 N}{3}}}\right),
$$

where $I_{s}(z)$ is the modified Bessel function of the first kind. 
Proof. By Corollary 12 with the change of variable $u=2 \sqrt{6 N} x$, we deduce

$$
\begin{aligned}
I^{\prime} & =\frac{1}{2 \sqrt{6 N}} \int_{-1}^{1} F_{a, b}\left(e^{\frac{\pi}{\sqrt{6 N}}(-1+i u)}\right) e^{\pi \sqrt{\frac{N}{6}}(1-i u)} d u \\
& =\frac{1}{2 \sqrt{6 N}} \int_{-1}^{1}\left[\frac{b}{4 \sqrt{2 \pi}}\left(\frac{\pi(1-i u)}{\sqrt{6 N}}\right)^{3 / 2} e^{\pi \sqrt{\frac{N}{6}}\left(\frac{1}{1-i u}+(1-i u)\right)}+\mathcal{O}\left(N^{-5 / 4} e^{2 \pi \sqrt{\frac{N}{6}}}\right)\right] d u \\
& =\frac{b}{4 \sqrt{2 \pi}}\left(\frac{\pi}{\sqrt{6 N}}\right)^{5 / 2} P_{3 / 2}+\mathcal{O}\left(N^{-7 / 4} e^{\pi \sqrt{\frac{2 N}{3}}}\right),
\end{aligned}
$$

where

$$
P_{s}:=\frac{1}{2 \pi i} \int_{1-i}^{1+i} v^{s} e^{\pi \sqrt{\frac{N}{6}}\left(v+\frac{1}{v}\right)} d v .
$$

Lemma 4.2 in [4] shows that

$$
P_{s}=I_{-s-1}\left(\pi \sqrt{\frac{2 N}{3}}\right)+\mathcal{O}\left(e^{\frac{3 \pi}{2} \sqrt{\frac{N}{6}}}\right)
$$

which completes the proof.

The next proposition shows that the second integral $I^{\prime \prime}$ is smaller than the error term in the main asymptotic formula of $I^{\prime}$.

Proposition 15. As $N \rightarrow \infty$,

$$
\left|I^{\prime \prime}\right| \ll e^{2 \pi \sqrt{\frac{N}{6}}-\frac{\sqrt{6 N}}{5 \pi}}
$$

Proof. By Corollary 13,

$$
\left|I^{\prime \prime}\right| \leqslant \int_{\frac{1}{2 \sqrt{6 N}} \leqslant|x| \leqslant \frac{1}{2}}\left|F_{a, b}\left(e^{-\pi \sqrt{\frac{N}{6}}+2 \pi i x}\right) e^{\pi \sqrt{\frac{N}{6}}-2 \pi i N x}\right| d x \ll e^{\pi \sqrt{\frac{N}{6}}} e^{\pi \sqrt{\frac{N}{6}}-\frac{\sqrt{6 N}}{5 \pi}} .
$$

In summary, we have obtained the following asymptotic formula for the coefficient $\alpha_{a, b}(N)$.

Corollary 16. For an positive integer $a$ and an integer $b$ with $a+b>0$ and $a \equiv b$ $(\bmod 2)$, as $N \rightarrow \infty$,

$$
\alpha_{a, b}(N)=b \frac{\pi^{2}}{24 \sqrt[4]{24}} N^{-5 / 4} I_{-5 / 2}\left(\pi \sqrt{\frac{2 N}{3}}\right)+\mathcal{O}\left(N^{-7 / 4} e^{\pi \sqrt{\frac{2 N}{3}}}\right) .
$$

Now, we are ready to prove Theorem 5. Since

$$
I_{s}(z) \sim \frac{e^{z}}{\sqrt{2 \pi z}}
$$


the first part of Theorem 5 follows immediately from Corollary 16.

For the second part of Theorem 5, note that

$$
\sum_{n=0}^{\infty} \alpha_{2 k, 0}(n) q^{n}=\frac{\left(q^{k} ; q^{2 k}\right)_{\infty}^{2}\left(q^{2 k} ; q^{2 k}\right)_{\infty}}{(q ; q)_{\infty}}
$$

where we set $a=2 k>2$ and have applied Jacobi's triple product identity [1, Theorem 2.8]. From this, we deduce that

$$
\begin{aligned}
\frac{\left(q^{k} ; q^{2 k}\right)_{\infty}^{2}\left(q^{2 k} ; q^{2 k}\right)_{\infty}}{(q ; q)_{\infty}} & =\frac{\left(q^{k} ; q^{2 k}\right)_{\infty}^{2}\left(q^{2 k} ; q^{2 k}\right)_{\infty}^{3}}{(q)_{\infty}\left(q^{2 k} ; q^{2 k}\right)_{\infty}^{2}} \\
& =\frac{\left(q^{k} ; q^{k}\right)_{\infty}^{2}}{(q ; q)_{\infty}\left(q^{2 k} ; q^{2 k}\right)_{\infty}} \\
& =\frac{\left(q^{k} ; q^{k}\right)_{\infty}^{k}}{(q ; q)_{\infty}\left(q^{k} ; q^{k}\right)_{\infty}^{k-2}\left(q^{2 k} ; q^{2 k}\right)_{\infty}}
\end{aligned}
$$

Since $\frac{\left(q^{k} ; q^{k}\right)_{\infty}^{k}}{(q ; q)_{\infty}}$ is a generating function for the number of $k$-core partition, it is clear that $q$-expansion of the above infinite product is nonnegative. By the result of A. Granvile and $\mathrm{K}$. Ono [6], we know that there is a $k$-core partition of $n$ if $k \geqslant 4$. Thus, when $k \geqslant 4$, the $q$-expansion of (2) has positive coefficients. When $k=3$, since the partitions 1 and $1+1$ are 3-core partitions and since $\frac{1}{\left(q^{3} ; q^{3}\right)_{\infty}}=\sum_{n=0}^{\infty} p(n) q^{3 n}$, the coefficients of $q$-expansion of (2) are always positive. Finally, when $k=2$, we see that (2) is

$$
\frac{\left(q^{2} ; q^{2}\right)_{\infty}^{2}}{(q ; q)_{\infty}\left(q^{4} ; q^{4}\right)_{\infty}}
$$

Note that the partitions $1,1+2$, and $1+2+3$ are 2 -core partitions and $\frac{1}{\left(q^{4} ; q^{4}\right)_{\infty}}=$ $\sum_{n=0}^{\infty} p(n) q^{4 n}$. Therefore, the $n$-th coefficient of $q$-expansion has positive coefficient provided $n \equiv 0,1,3,6$. Hence, the $q$-expansion of (2) has positive coefficients except $n=2$. This completes the proof of the second part of Theorem 5.

\section{Acknowledgments}

The authors would like to thank Bruce Berndt, Jehanne Dousse, and the referee for their careful reading and comments on an earlier version of the paper.

\section{References}

[1] G.E. Andrews, The theory of partitions, Addison-Wesley, Reading, MA, 1976; reissued by Cambridge University Press, Cambridge, 1984.

[2] G. E. Andrews, Concave compositions. Electronic Journal of Combinatorics 18 (2011), P6. 
[3] B. C. Berndt, B. Kim, Asymptotic expansions of certain partial theta function, Proc. Amer. Math. Soc. 139 (2011), 3779-3788.

[4] K. Bringmann, K. Mahlburg, Asymptotic inequalities for positive crank and rank moments, Trans. Amer. Math. Soc. 366 (2014), 1073-1094.

[5] K. Bringmann, J. Dousse, On Dyson's crank conjecture and the uniform asymptotic behavior of certain inverse theta functions, Trans. Amer. Math. Soc., to appear.

[6] A. Granville, K. Ono, Defect zero p-blocks for finite simple groups, Trans. Amer. Math. Soc. 348 (1996), 331-347.

[7] A. Ingham, A Tauberian theorem for partitions, Ann. of Math. 42 (1941), 1075-1090.

[8] S. Jo and B. Kim, On asymptotic formulas for certain q-series involving partial theta functions, Proc. Amer. Math. Soc., to appear.

[9] B. Kim, On the subpartitions of the ordinary partitions, Ramanujan J.(Special issue for G.E. Andrews' 70th birthday) 23 (2010), 159-167.

[10] L.W. Kolitsch, Rogers-Ramanujan subpartitions and their connections to other partition, Ramanujan J., 2008, 163-167.

[11] S. Ramanujan, The lost notebook and other unpublished papers, Narosa, New Delhi, 1988.

[12] D. Zagier, The Mellin transform and other useful analytic techniques, Appendix to E. Zeidler, Quantum Field Theory I: Basics in Mathematics and Physics. A Bridge Between Mathematicians and Physicists Springer-Verlag, Berlin-Heidelberg-New York (2006), 305-323. 